\title{
Optical Assessment of Tear Glucose by Smart Biosensor based on Nanoparticle Embedded
}

\section{Contact Lens}

Hee-Jae Jeon ${ }^{1,2}$, Sooyeon Kim ${ }^{1}$, Sijin Park ${ }^{3,8}$, In-Kyung Jeong', Jaheon Kang', Young Ro Kim ${ }^{5,6}$, Dong Yun Lee $e^{3,8} \$$, and Euiheon Chung ${ }^{1,7} \ddagger$

${ }^{1}$ Department of Biomedical Science and Engineering, Gwangju Institute of Science and Technology (GIST), Gwangju, 61005, Republic of Korea

${ }^{2}$ Weldon School of Biomedical Engineering, Purdue University, West Lafayette, Indiana 47907, United States

${ }^{3}$ Department of Bioengineering, College of Engineering, and BK FOUR Biopharmaceutical Innovation Leader for Education and Research Group, and Institute of Nano Science \& Technology (INST), Hanyang University, Seoul 04763, Republic of Korea

${ }^{4}$ Department of Endocrinology and Metabolism, Kyung Hee University Hospital at Gangdong, Kyung Hee University School of Medicine, Seoul 02447, Republic of Korea ${ }^{5}$ Department of Radiology, Harvard Medical School, Boston, Massachusetts 02115, United States

${ }^{6}$ Athinoula A. Martinos Center for Biomedical Imaging, Massachusetts General Hospital, Charlestown, Massachusetts 02129, United States

${ }^{7}$ AI Graduate School, Gwangju Institute of Science and Technology (GIST), Gwangju, 61005, Republic of Korea

${ }^{8}$ Elixir Pharmatech Inc., Seoul 04763, Republic of Korea 
Animal preparation for tear glucose monitoring. The research was carried out with eight mice (C57BL/6) and eight Diabetic transgenic mice (BKS.Cg-m+/Lepr/J_CRJ, C57BL/6) under the rules of the Institute Animal Care and Use Committee (IACUC) of the Gwangju Institute of Science and Technology (Protocol Number: GIST-2019-015). Three experimental groups were prepared: 1) Awake group, 2) Inhalant anesthesia group using isoflurane (2 3\% at the flow rate of $0.5-0.71 / \mathrm{min}$ ), and 3) Injectable anesthesia group using Zoletil $(60 / 10 \mathrm{mg} / \mathrm{kg}$ body weight). Before measuring tear glucose concentration, the blood glucose levels were measured with a blood glucometer (Accu-Chek Performa, Roche Diagnostics, Switzerland) by pricking the mouse tail after 8 hours of fasting as described in Supporting information Table S1. We prepared two types of chambers between the anesthesia group (Isoflurane and Zoletil) and the awake group (normal and transgenic mice). For the awake group, a custom-made body holder was created with a 3D printer to restrain the mouse body and head (Fig. S1a). For the anesthesia group, a commercial mouse head holder (SA-8, Narishige, Narishige Scientific Instrument Lab, Japan) was used. A heating pad was used to keep the body temperature (Braintree Scientific, Deltaphase Isothermal Pad, model: 39DP).

The human tear collection. Human tears were collected from eight volunteers (4 diabetic patients and 4 healthy subjects) of both genders between 20 and 50 years old; see Supporting information Table S2. For inducing the high glucose level, the tear samples were collected at 2 hours after eating food. We used a microcapillary tube (Blaubrand IntraEND, Wertheim, Germany) to collect human tears without any stimulation. The glass tube was carefully rested in the lateral tear meniscus to minimize contact with the bulbar conjunctiva or the lid margin, as depicted in Figure 5a. The same clinician always collected tears without topical anesthesia. The collection time was limited to a maximum of $30 \mathrm{~min}$, with collection volumes between $5 \sim 10 \mu \mathrm{L}$. All clinical samples were transferred to polypropylenes tubes immediately and stored under $-70{ }^{\circ} \mathrm{C}$ until further processing.

\section{Image Processing algorithm (Figure 2)}

Phase 1. Automatically finding the NECL center. Once the original colorimetric NECL was captured, our algorithm finds the circles with a diameter of $2 \mathrm{~mm}$. A low threshold value was subtracted throughout the NECL image to remove the background using a flood-filling 
function [26]. After successfully extracting the NECL, the edge and the center of the NECL were determined using the Sobel function and centroid in MATLAB (Mathworks, Natick, MA). Then the rectangular ROI $(150 \times 150$ pixels $)$ from each image was selected based on centroid positions.

Phase 2. Glare subtraction. Glare is the saturated region in an image when the light is strongly reflected on the NECL surface resulting in loss of information. Thus, we developed an algorithm to delineate the glare area and subtract it. The glare region was excluded for further analysis within the ROI.

Phase 3. Fractional RGB calculation. We defined fractional RGB intensities with the ROI image, which comprises three RGB channels as follows:

$\mathrm{r}=\mathrm{R} /(\mathrm{R}+\mathrm{G}+\mathrm{B}), \quad \mathrm{g}=\mathrm{G} /(\mathrm{R}+\mathrm{G}+\mathrm{B}), \quad \mathrm{b}=\mathrm{b} /(\mathrm{R}+\mathrm{G}+\mathrm{B})$

, where R, G, and B are the mean values of each red, green, and blue channel of the ROI image. The mean and standard deviation of fractional $R, G$, and $B$ intensities regarding the tear glucose concentration were calculated.

Phase 4. Tear glucose estimation. The estimated tear glucose concentrations were calculated by the arithmetic mean of the three channels with equation (2)

Tear glucose $=(\mathrm{Cr}+\mathrm{Cg}+\mathrm{Cb}) / 3$

, where $\mathrm{Cr}, \mathrm{Cg}$, and $\mathrm{Cb}$ are tear glucose concentration through fractional red, green, and blue intensity, respectively. The overall process of a movie is shown on supplementary material (Movie S2). 
Movie S1. The NECL was automatically tracking by finding the center position even in the presence of image shaking. The right side movie shows the original image frames, and the left side movie shows the continuously tracked image frames of NECL position. 


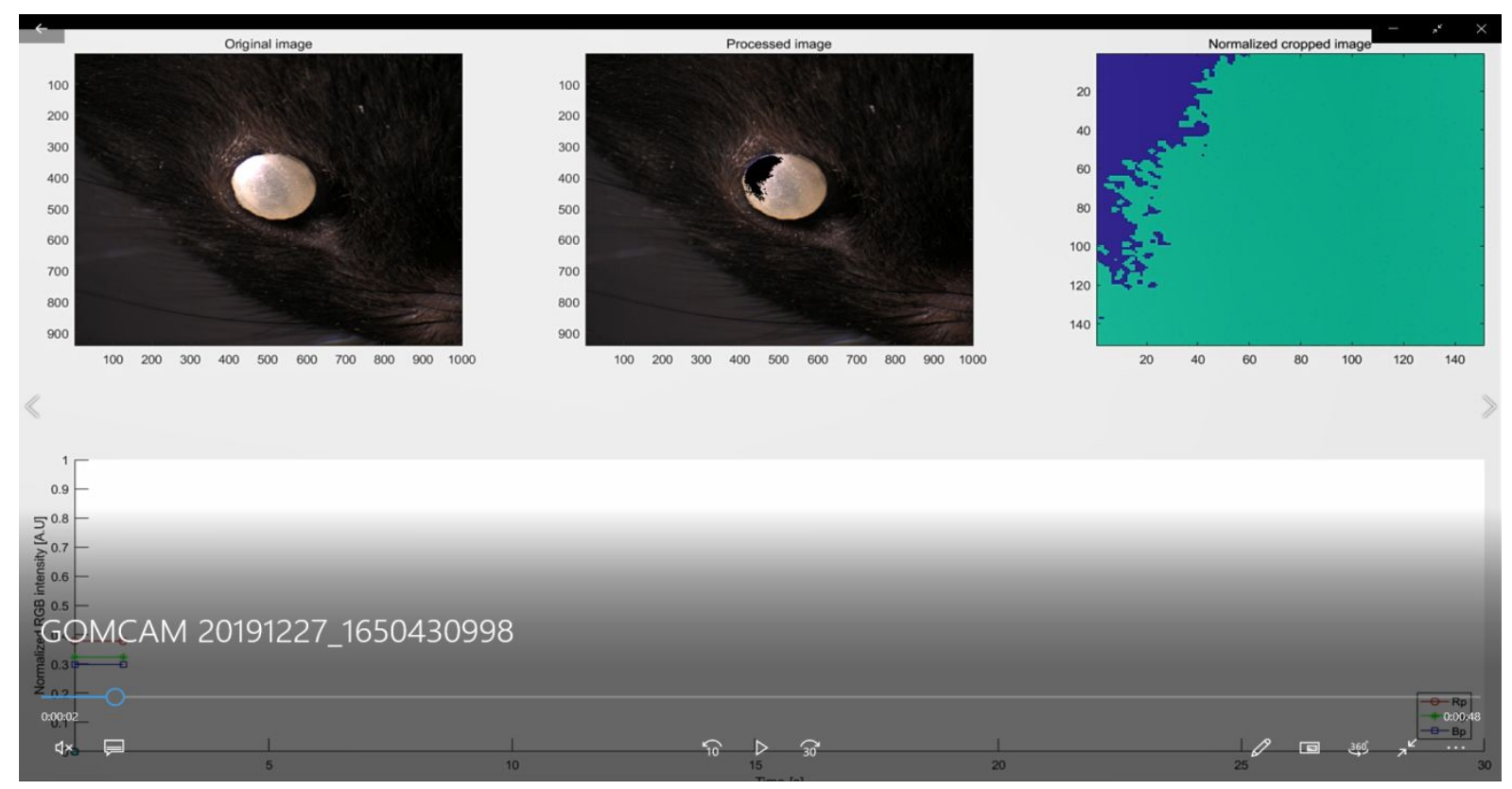

Movie S2. Overall working flow to calculate fractional $\mathbf{r}, \mathbf{g}, \mathbf{b}$ intensities. NECL color

change was monitored by calculating fractional $\mathrm{r}, \mathrm{g}, \mathrm{b}$ intensities and automatically removed image frames if there are image shaking due to eye blinking, body fluctuation, and breathing. Analysis movie combined with original video, processed video, and fractional r, g, b intensity distribution. 

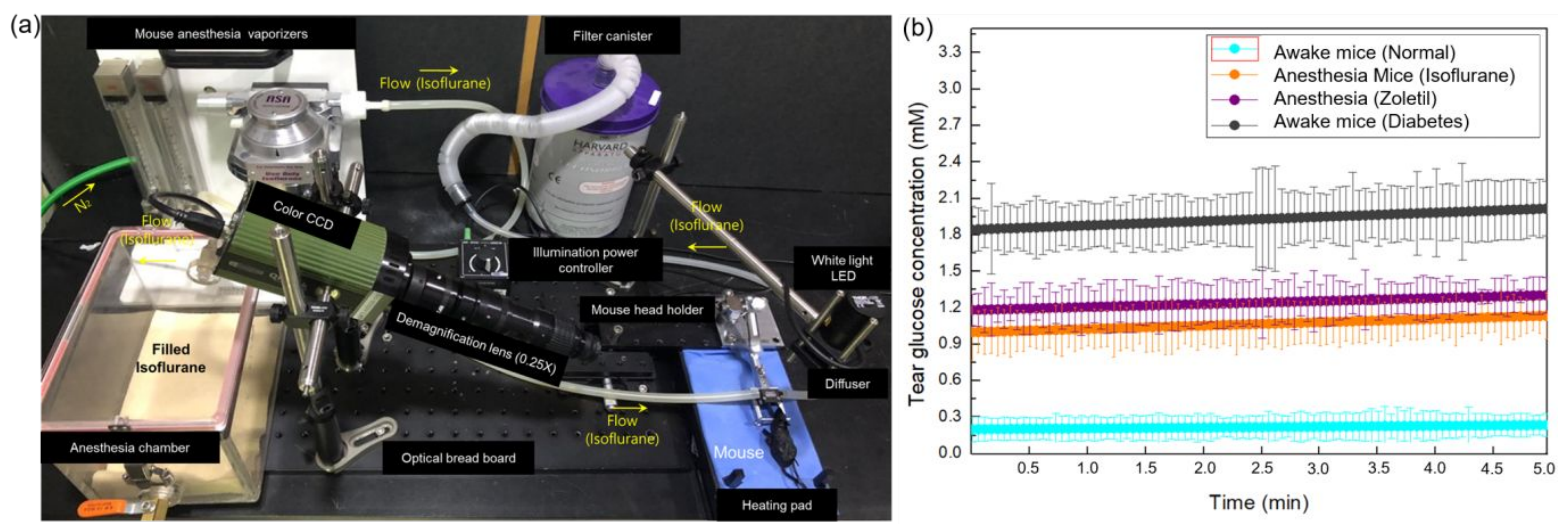

Figure S1. The optical monitoring system to estimate tear glucose concentration for

mice. (a) The experimental configuration consisted of an anesthesia chamber, color CCD camera, demagnification zoom lens (45X), white light LED, LED driver, diffuser filter chamber, heating pad, and head holder. The color change of nanoparticle embedded contact lens was monitored for $5 \mathrm{~min}$ after put mouse eye. (b) We continuously estimated tear glucose concentration for 5 min in normal, anesthesia (isoflurane, Zoletil), and diabetic mice. 

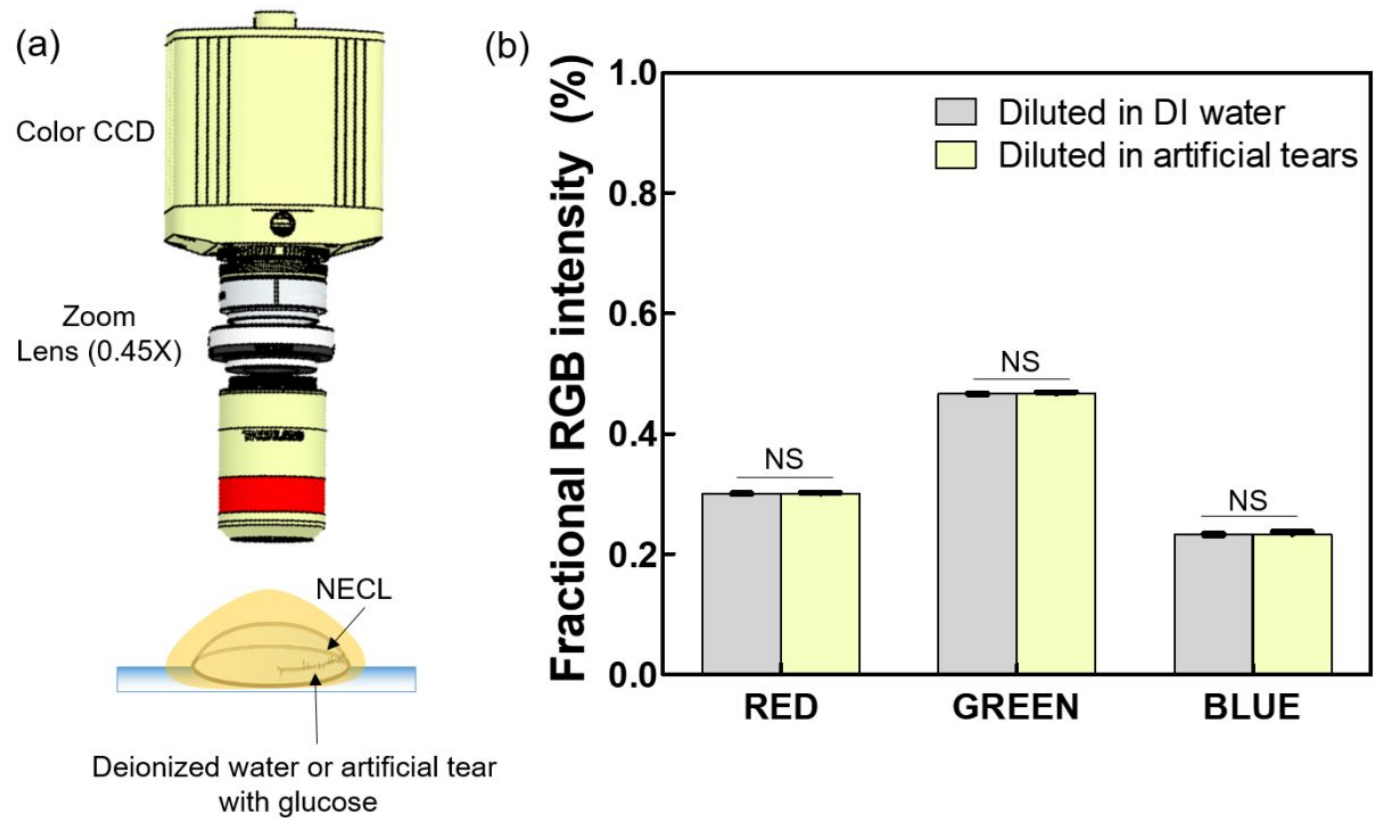

Figure S2. The comparison of fractional $\mathbf{r}, \mathbf{g}, \mathrm{b}$ intensities between artificial tear solution and deionized water. (a) The optical monitoring system consisted of color CCD, a demagnification zoom lens with a $0.45 \mathrm{X}$. (b) We monitored nanoparticle embedded contact lens (NECL) color change after soaked each solution. $100 \mu \mathbf{~ o f ~ D I ~ w a t e r ~ a n d ~ a r t i f i c i a l ~ t e a r ~}$ solutions, including $1 \mathrm{mM}$ glucose, were prepared to confirm the difference $(\mathrm{n}=10)$. 


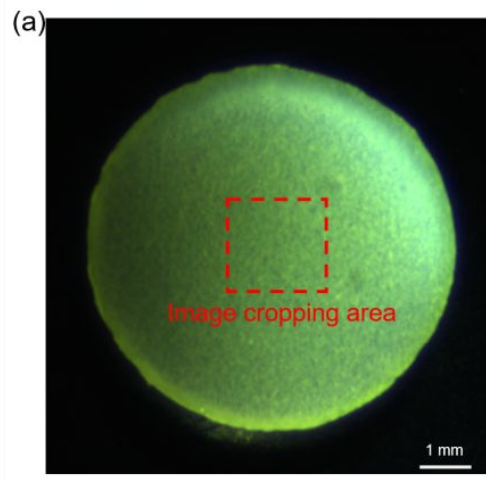

(b)

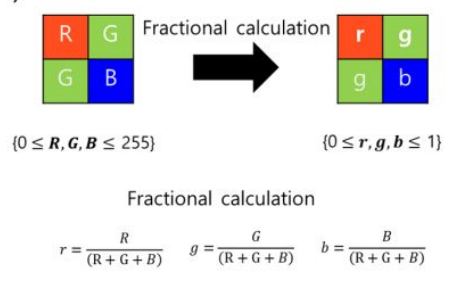

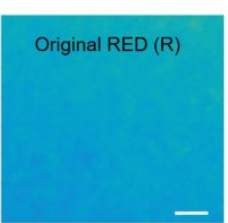

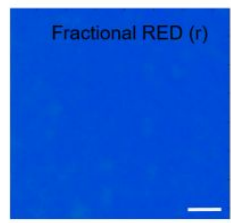

(c)
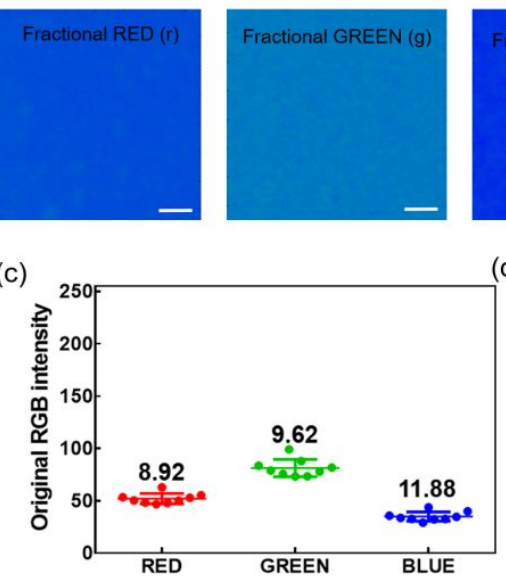
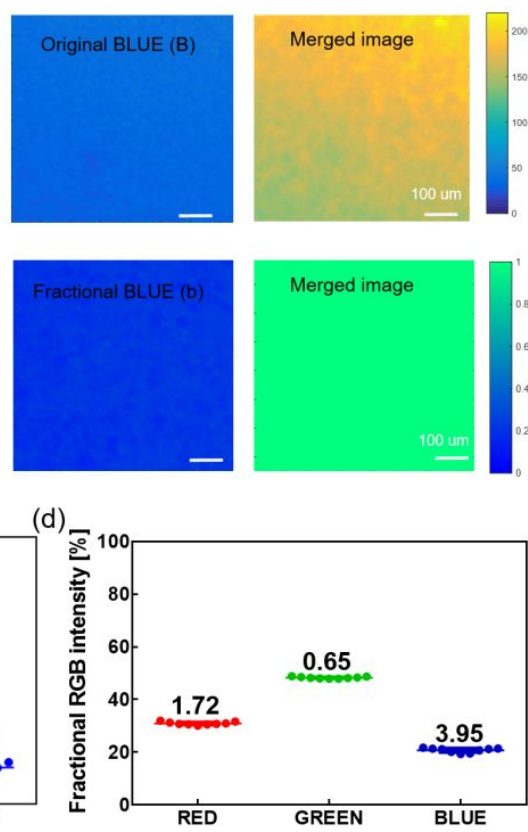

Figure S3. The comparison of image processing between the original and fractional channels. (a) RGB distribution images acquired from the original and fractional channels. The fractional $r, g$, b channels were calculated by the original $R, G$, and B values from the image cropping area $(0.7 \times 0.7)$ and show the calculation equation $(b \sim d)$ shows the averaged intensity distribution from the original RGB channel and the fraction RGB channel. The relative variability was calculated by the standard deviation/mean of $\mathrm{r}, \mathrm{g}, \mathrm{b}$ channels. 


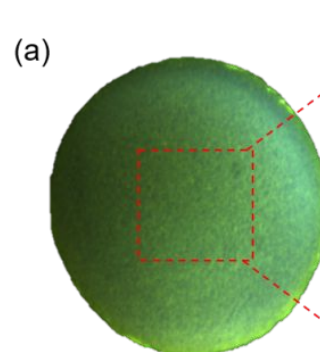

Original NECL

(b)

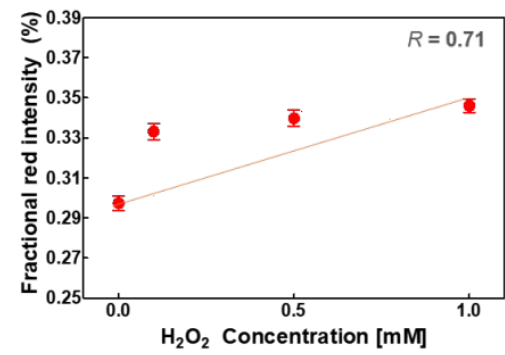

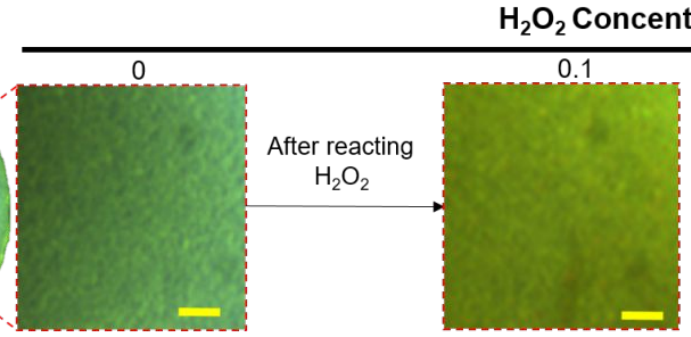

(c)

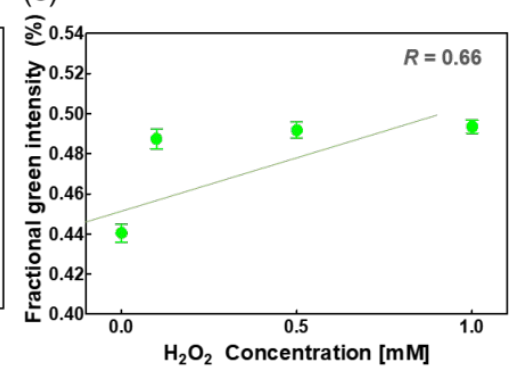

(d)

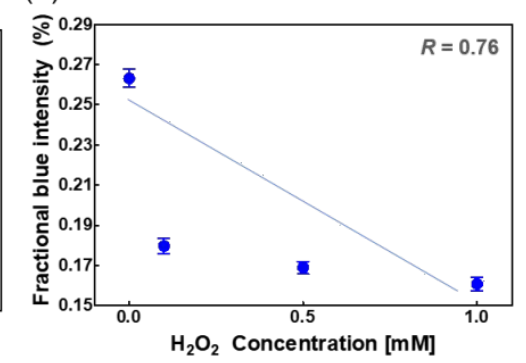

Figure S4. The confirmation of NECL color change by comparing fractional $r, g, b$ intensities at different $\mathrm{H}_{2} \mathrm{O}_{2}$ concentrations. (a) The color of NECL turns yellowish after reacting with $\mathrm{H}_{2} \mathrm{O}_{2}$. $(\mathrm{B} \sim \mathrm{D})$ We plotted fractional $\mathrm{r}, \mathrm{g}, \mathrm{b}$ intensities following reaction with $\mathrm{H}_{2} \mathrm{O}_{2}$ for $1 \mathrm{~min}$. The red, green, blue line: fractional red, green, blue intensity linear regression, Scale bar size: $200 \mu \mathrm{m},(\mathrm{n}=10)$. 

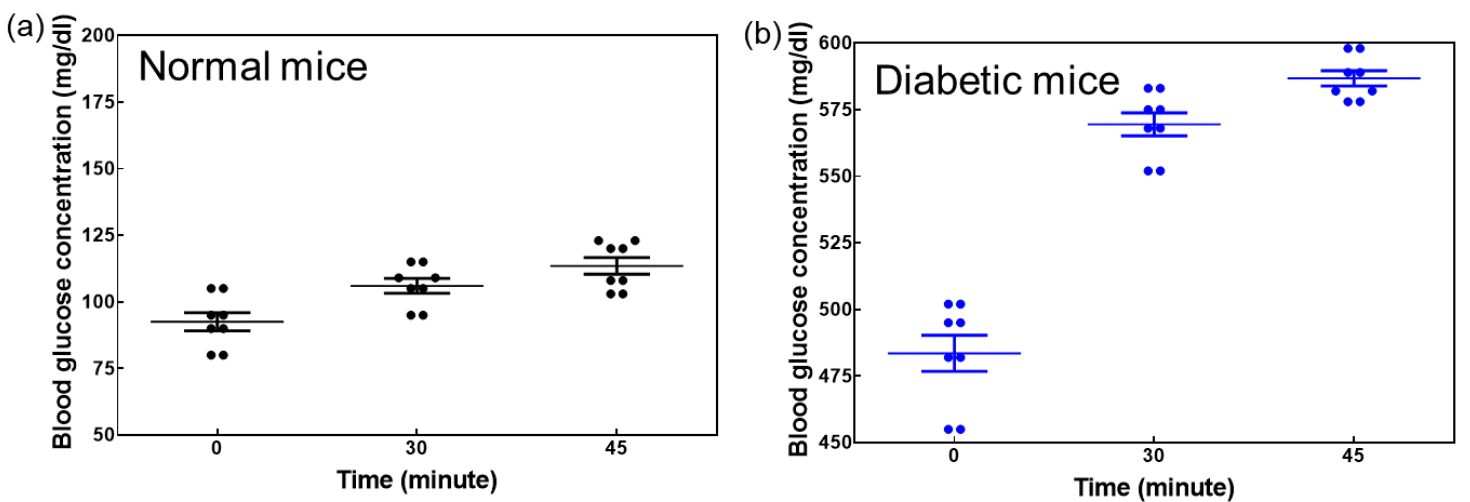

Figure S5. Measurement of blood glucose concentration with normal and transgenic

mice. Awake mice (normal and diabetic mice) retained a custom-made mouse holder to reduce the body motion for the quantitative measurement of tear glucose concentration. Blood glucose concentration was slightly increased in normal mice (a) than transgenic diabetic mice (b). (black dots: normal mice, blue dots: transgenic diabetic mice) $(n=8)$ 
(a)

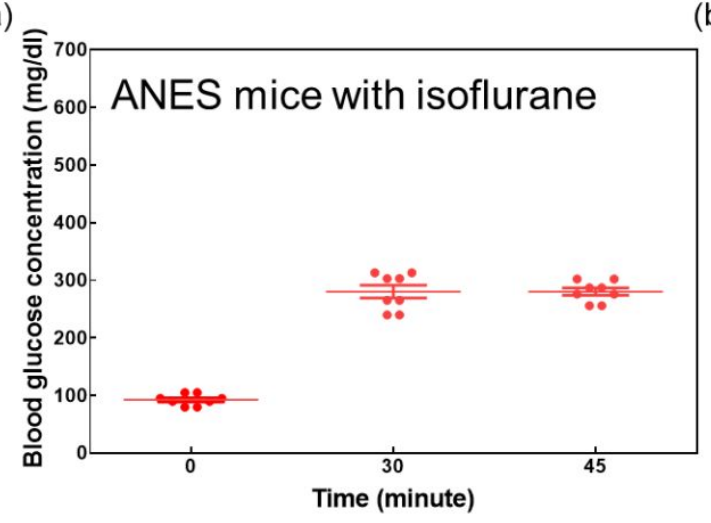

(b)

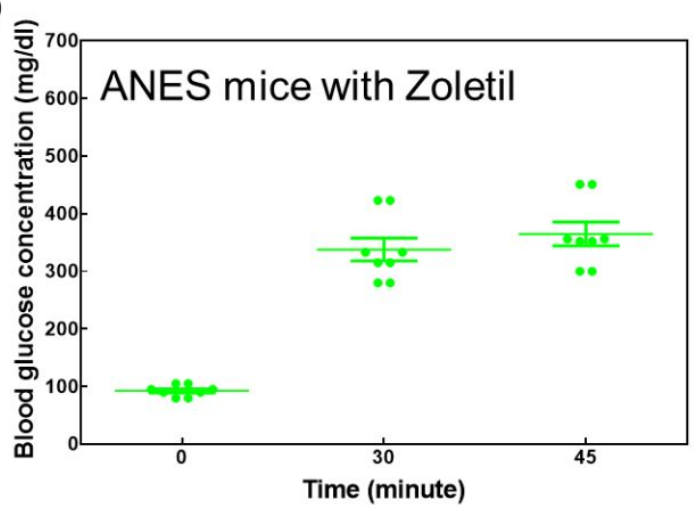

Figure S6. Measurement of blood glucose concentration with anesthesia mice (isoflurane and Zoletil). The blood glucose concentration increased until 30 minutes in the anesthesia mice with isoflurane (a) and Zoletil (b). The blood glucose concentration was not changed after 30 minutes. (red dots: anesthesia mice with isoflurane, green dots: anesthesia mice with Zoletil), ANES; Anesthesia, $(n=8)$. 
(a)
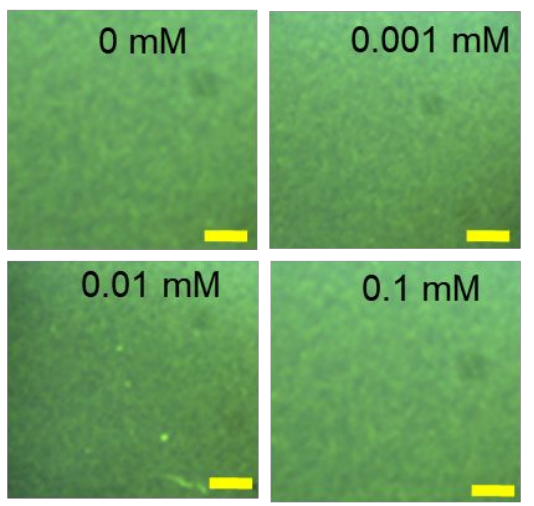

(c)

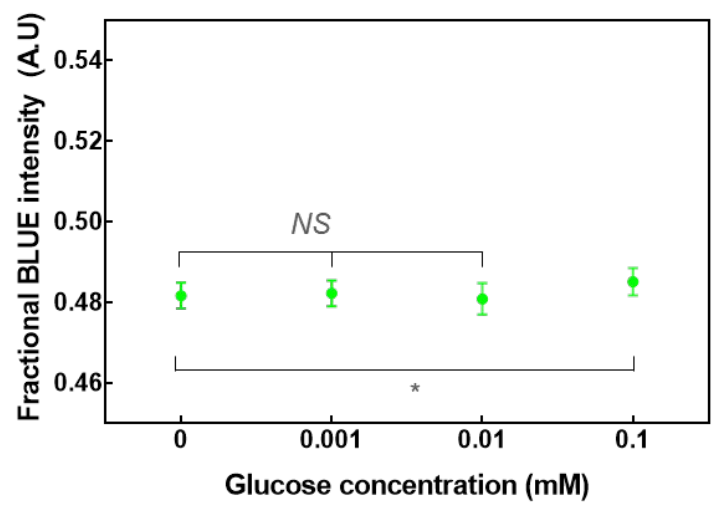

(b)

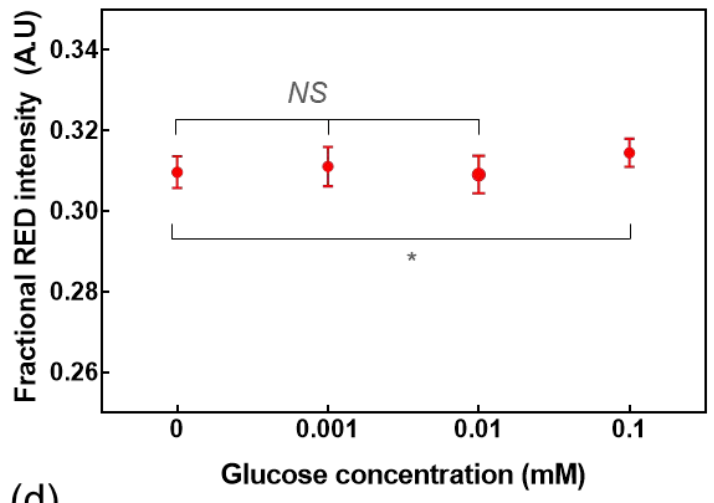

(d)

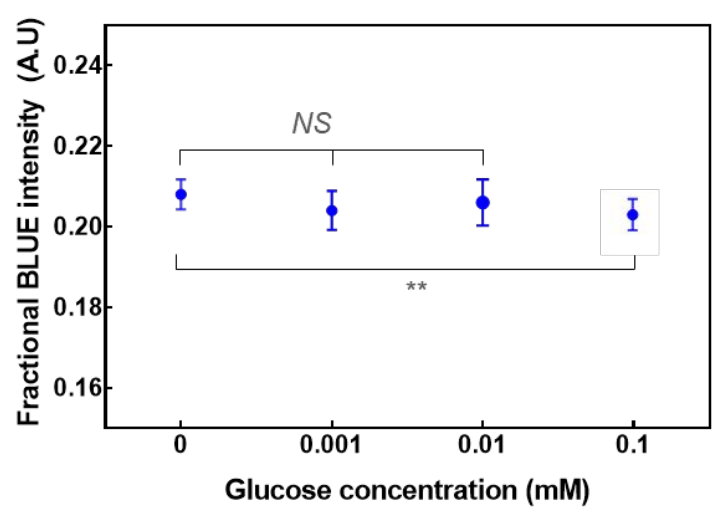

Figure S7. Measurement of the limit of detection (LOD) at the low glucose concentrations. The glucose solutions with different concentrations $(0,0.001,0.01$, and 0.1 $\mathrm{mM}$ ) were prepared for the measurement limit of detection (LOD). The fractional RGB intensities were calculated at the low levels of different glucose concentrations $(n=6)$. $*$ indicates $\mathrm{p}<0.05, * *$ indicates $\mathrm{p}<0.01$, and NS indicates $\mathrm{p}>0.5$. 
(a)

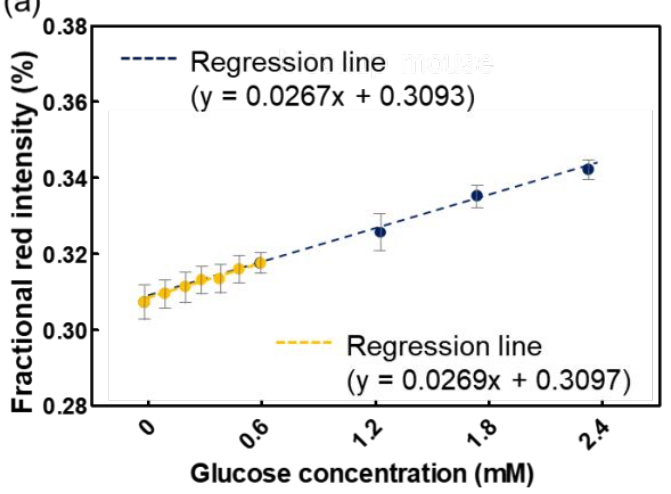

(c)

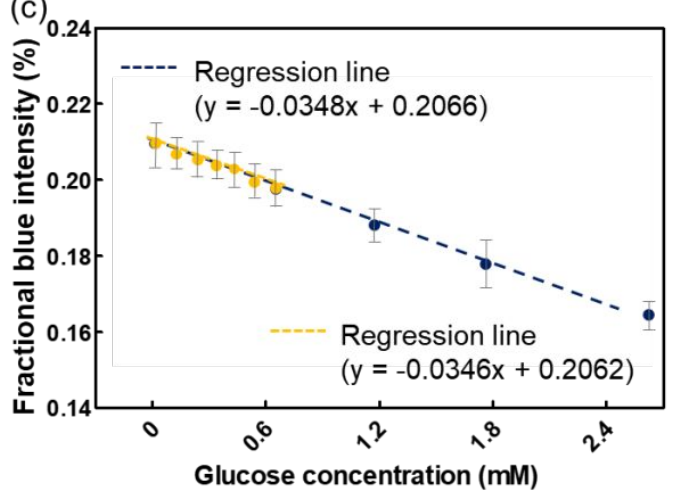

(b)

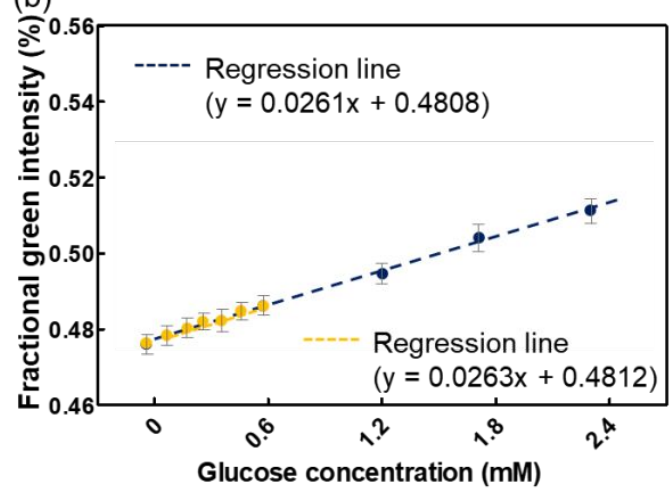

(d)

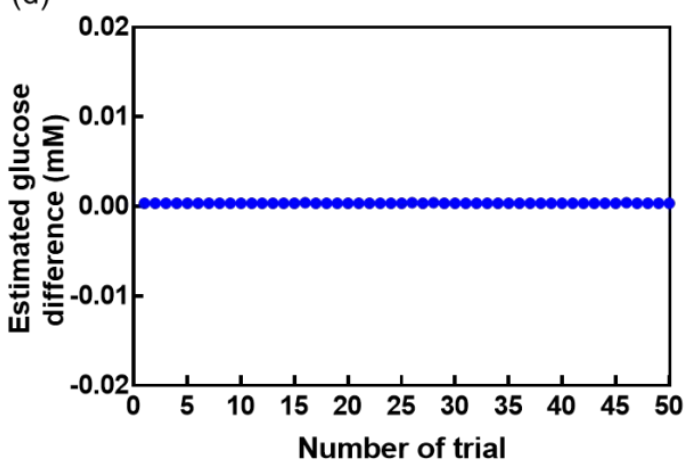

Figure S8. The estimation of curve fitting at the low glucose concentration with linear

regression. $(\mathrm{a} \sim \mathrm{c})$. The linear regression line $(\mathrm{y}=\mathrm{ax}+\mathrm{b})$ was measured between (1) low glucose concentration $(0 \sim 0.6 \mathrm{mM})$ with $0.1 \mathrm{mM}$ interval, and (2) high glucose concentration $(0 \sim 2.4 \mathrm{mM})$ with $0.6 \mathrm{mM}$ interval. Yellow dotted line and dot points show the low glucose concentration measurement, and dark blue show the high glucose concentration measurement results. (d) The estimated glucose difference from two linear regression lines was measured in 50 trials. 


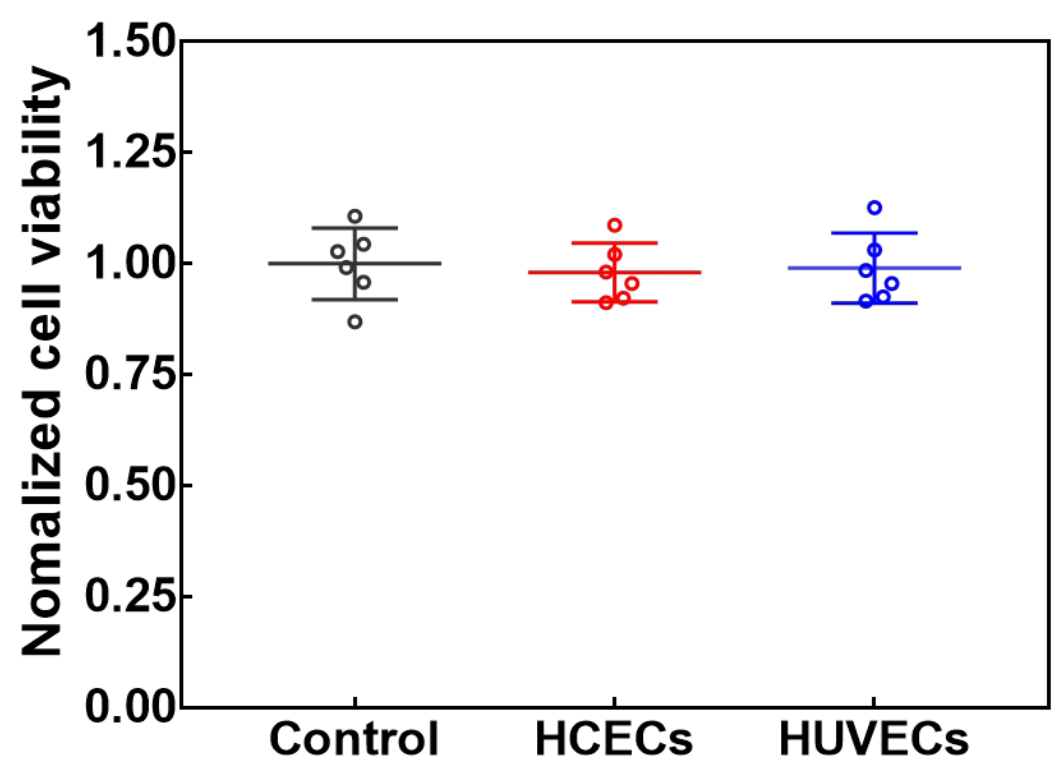

Figure S9. The cytotoxicity of the NECLs. The cell viability of control, human corneal epithelial cells (HCECs), and human umbilical vein endothelial cells (HUVECs) against NECLs were measured after 24 hours. The mean of normalized cell viability of control, HCECs, and HUVECs were $1 \pm 0.08$ and $0.98 \pm 0.065$, and $0.99 \pm 0.08$, respectively $(n=6)$ without any statistical differences. 


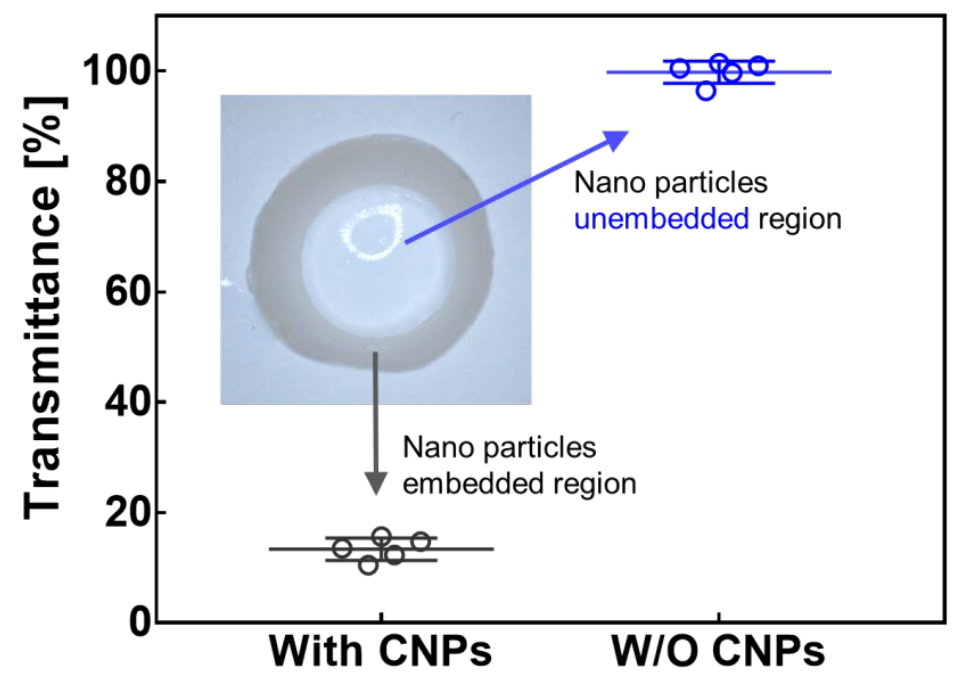

Figure S10. The transmittance measurement of center-transparent NECLs. We fabricated center-transparent NECLs with a doughnut shape to improve visibility and measured the transmittance in each region. The central region had a high transmittance close to $100 \%$ and the nanoparticle embedded region had a poor transmittance below $20 \%$. 
Table S1. Gender, age, and diabetes status of mice used for tear and blood glucose measurements.

\begin{tabular}{|c|c|c|c|c|c|}
\hline Number & Sex & Weeks & Status (Animal test) & Measured eye & Fasting time (hour) \\
\hline 1 & Female & $10 \sim 12$ & Transgenic diabetic mouse & Left & $<8$ \\
\hline 2 & & & & Right & \\
\hline 3 & Female & $10 \sim 12$ & Transgenic diabetic mouse & Left & $<8$ \\
\hline 4 & & & & Right & \\
\hline 5 & Male & $10 \sim 12$ & Transgenic diabetic mouse & Left & $<8$ \\
\hline 6 & & & & Right & \\
\hline 7 & Male & $10 \sim 12$ & Transgenic diabetic mouse & Left & $<8$ \\
\hline 8 & & & & Right & \\
\hline 9 & Female & $10 \sim 12$ & Normal mouse & Left & $<8$ \\
\hline 10 & & & & Right & \\
\hline 11 & Female & $10 \sim 12$ & Normal mouse & Left & $<8$ \\
\hline 12 & & & & Right & \\
\hline 13 & Male & $10 \sim 12$ & Normal mouse & Left & $<8$ \\
\hline 14 & & & & Right & \\
\hline 15 & Male & $10 \sim 12$ & Normal mouse & Left & $<8$ \\
\hline 16 & & & & Right & \\
\hline
\end{tabular}


Table S2. Gender, age, and diabetes status of human subjects used for tear and blood glucose measurements

\begin{tabular}{|c|c|c|c|c|c|}
\hline Number & Sex & Age & Status (Clinical test) & Measured eye & Fasting time (hour) \\
\hline 1 & Female & 49 & Diabetic & Left & 2 \\
\hline 2 & & & & Right & \\
\hline 3 & Female & 58 & Diabetic & Left & 2 \\
\hline 4 & & & & Right & \\
\hline 5 & Female & 56 & Non-diabetic & Left & 2 \\
\hline 6 & & & & Right & \\
\hline 7 & Male & 44 & Non-diabetic & Left & 2 \\
\hline 8 & & & & Right & \\
\hline 9 & Female & 59 & Diabetic & Left & 2 \\
\hline 10 & & & & Right & \\
\hline 11 & Female & 28 & Non-diabetic & Left & 2 \\
\hline 12 & & & & Right & \\
\hline 13 & Male & 33 & Non-diabetic & Right & 2 \\
\hline 14 & Male & 33 & Non-diabetic & Right & 2 \\
\hline 15 & Male & 39 & Non-diabetic & Right & 2 \\
\hline 16 & Male & 59 & Diabetic & Right & 2 \\
\hline
\end{tabular}

